\title{
Comunidades de aprendizaje
}

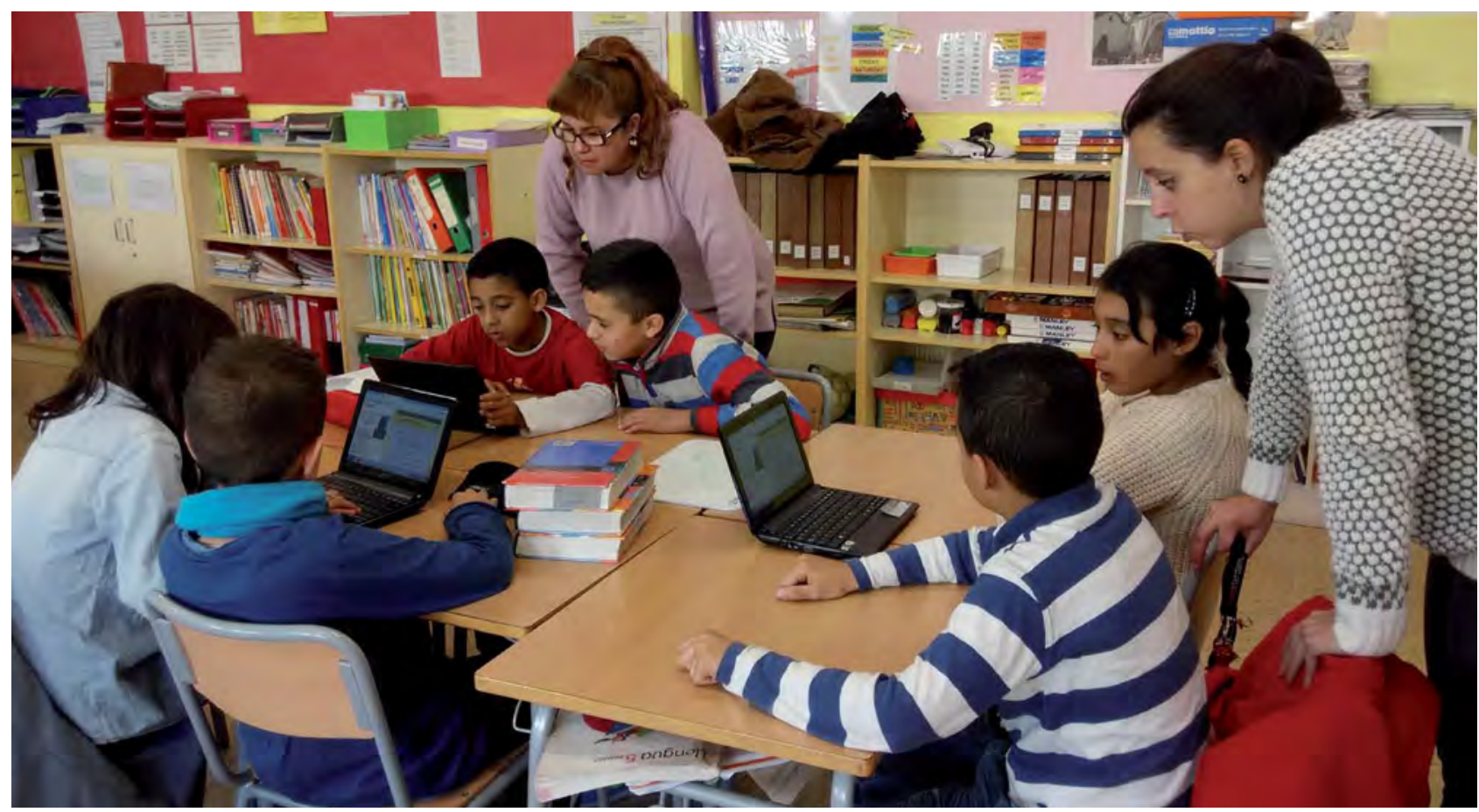

\section{Actuaciones para el éxito académico y la transformación educativa}

Las comunidades de aprendizaje nos invitan a pensar una escuela que basa sus actuaciones en evidencias científicas, que persigue el éxito académico de todo el alumnado y que promueve la cohesión social. Una escuela que traspasa las paredes del propio centro y que es capaz de transformar la realidad en la que se inscribe. En definitiva, nos abren las puertas a una comunidad que sueña la mejor educación para todo el alumnado, y que hace posible este sueño a partir de la implementación de

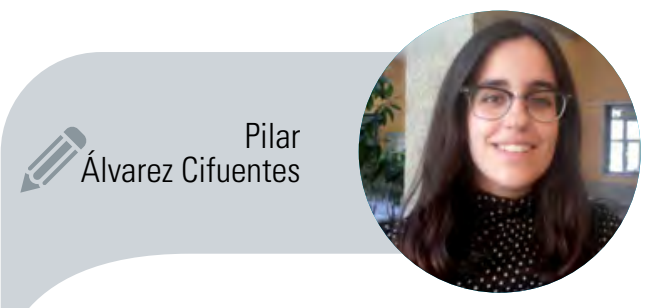

Facultad de Ciencias Sociales y Humanidades, Universidad Loyola Andalucía palvarez@uloyola.es actuaciones educativas de éxito.

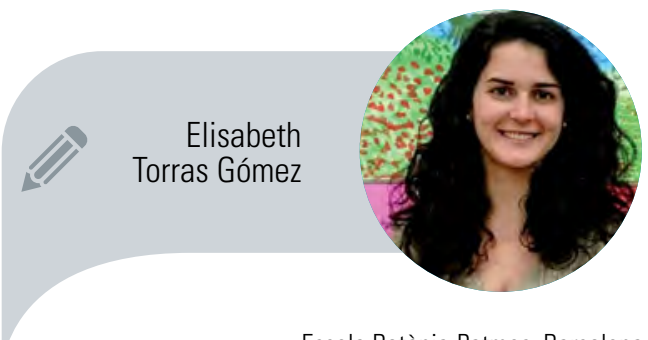


El reto de la mejora de resultados: de las ocurrencias a las evidencias

La mejora educativa es una de las principales preocupaciones para garantizar la inclusión social. En el plano europeo, la estrategia Europa 2020 sitúa entre una de sus cinco prioridades la disminución del abandono escolar temprano por debajo del $10 \%$ y el aumento de los niveles formativos de toda la población. En definitiva, se destaca la necesidad de avanzar en la consecución de la mejora de resultados educativos para toda la ciudadanía.

En lo que respecta al sistema educativo español, uno de los principales desafíos todavía pendientes es garantizar el éxito académico para todos: el alumnado español puntúa en matemáticas, lectura y ciencias por debajo, tanto de la media europea, como de la media de la OCDE. Resulta clave, por tanto, avanzar en la promoción de aquellas iniciativas educativas que están demostrando ser exitosas en la superación del fracaso escolar y que están conduciendo a promover el éxito educativo en todos los contextos.

Para poder avanzar en esta mejora de resultados es imprescindible superar los enfoques educativos basados en ocurrencias, es decir, aquellos que no se basan en las mejores evidencias científicas. Está claro que todo el profesorado quiere lo mejor para su alumnado, pero es importante tener en mente que nuestros alumnos se merecen sólo aquellas actuaciones que han demostrado ser eficaces a la hora de promover el éxito académico del alumnado y su máximo desarrollo en todos los niveles. Veamos un ejemplo para entender mejor esta idea: cuando estamos enfermos y necesitamos tratamiento queremos recibir el mejor posible, el que nos dé mayores garantías de éxito, en base a los resultados obtenidos en ensayos y otras investigaciones científicas. Difícilmente estaríamos de acuerdo con el médico, por muy buena voluntad y ganas que demuestre, si a pesar de lo que señala la comunidad científica nos prescribiera algo que no ha sido avalado. Así pues, el principio que

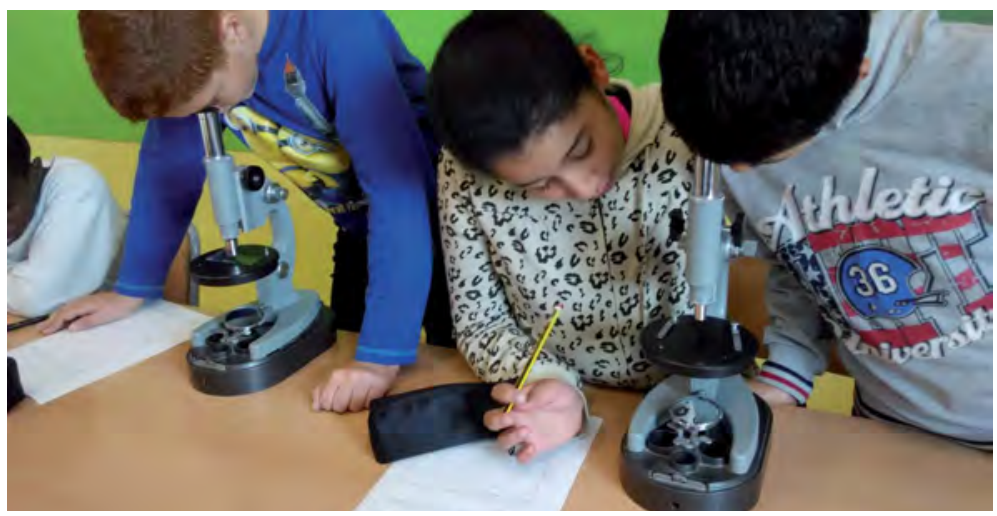

COMUNIDADES DE APRENDIZAJE SCHOOL AS "LEARNING COMMUNITIES"

"Comunidades de aprendizaje" es un proyecto basado en un conjunto de actuaciones educativas de éxito dirigidas a la transformación social y educativa. Este modelo educativo está en consonancia con las teorías cientíicas a nivel internacional que destacan dos factores claves para el aprendizaje en la actual sociedad: las interacciones y la participación de la comunidad.

\section{Nuestros alumnos se merecen sólo aquellas}

achuciones que han demostrado ser eficaces a la

hora de promover el éxilo académico del alumnado y

su máximo desarrollo en todos los niveles

lleva a los médicos a prescribir solamente aquellos tratamientos que hayan sido científicamente avalados y que nos parece tan evidente, aún no se está aplicando en su totalidad en el ámbito educativo. Por ello, con frecuencia en muchas aulas se está privando al alumnado de su derecho a acceder a las mejores actuaciones educativas disponibles.

\section{Comunidades de aprendizaje: evidencias científicas para la mejora de resultados de todo el alumnado}

En respuesta a los retos educativos que todavía tenemos por delante en el contexto español, son cada vez más las escuelas en nuestro país que se comprometen con la necesidad de exigir el máximo rigor científico en educación. Este compromiso es el motor que ha llevado a multitud de centros educativos a decidir- 


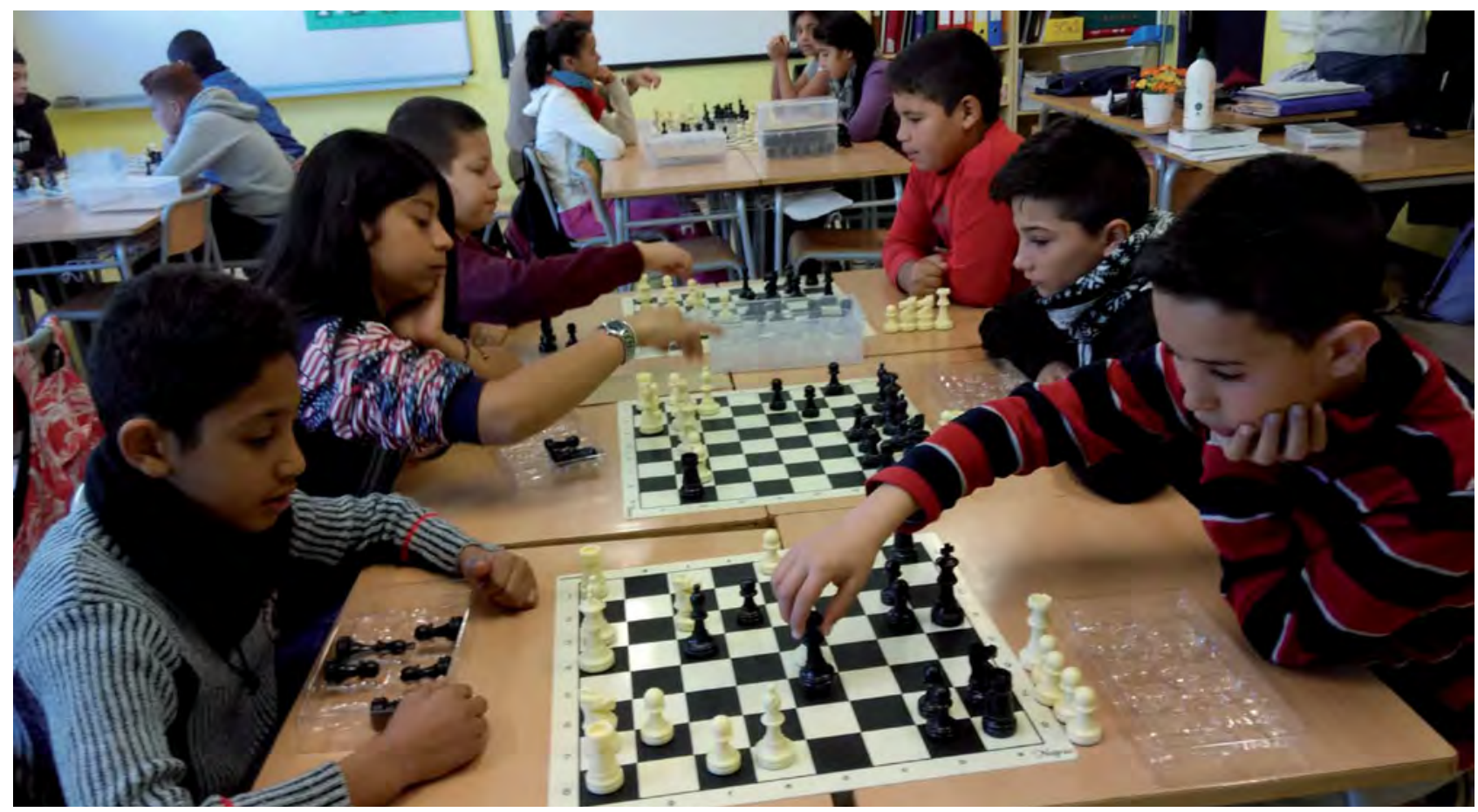

se a transformar su escuela en una comunidad de aprendizaje.

El proyecto "Comunidades de aprendizaje", con más de 20 años de trayectoria en la enseñanza obligatoria en España, es un proyecto basado en un conjunto de actuaciones educativas de éxito dirigidas a la transformación social y educativa. Este modelo educativo está en consonancia con las teorías científicas a nivel internacional que destacan dos factores clave para el aprendizaje en la actual sociedad: las interacciones y la participación de la comunidad. Para ello, las comunidades de aprendizaje se fundamentan en la concepción dialógica del aprendizaje, según la cual el conocimiento se construye a partir de las interacciones que compartimos con los demás. De este modo, en las escuelas que se organizan como comunidades de aprendizaje, familias, profesorado, alumnado y otros miembros de la comunidad se coordinan y colaboran para implementar actuaciones educativas de éxito, de modo que cada centro se nutre del bagaje que pueden aportarle todos los miembros de la comunidad y a la vez comparte con ellos la responsabilidad de la educación y el completo desarrollo de los niños. Este planteamiento implica aceptar que en la sociedad de la información no sólo es la escuela la que educa, sino que aprendemos en múltiples contextos y que las interacciones con los demás amplían y enriquecen nuestro conocimiento, por lo que el peso de la educación no puede recaer solamente sobre el profesorado. Así, desde esta nueva perspectiva, se pide la colaboración y participación de toda la comunidad a través de un diálogo igualitario, al que cada cual aporta el conjunto de experiencias y conocimientos que acumula en forma de inteligencia cultural, y donde la validez de las intervenciones yace en los argumentos que las sustentan y no en la posición de poder que ocupa la persona que las propone. De este modo, el papel de la escuela ya no se centra en transmitir y enseñar a reproducir, sino en promover el máximo número de interacciones entre el alumnado y la comunidad para facilitar la construcción y transformación de un conocimiento sólido que fomente un espíritu crítico, que garantice los máximos resultados académicos y que sea creador de sentido, a la vez que se desarrollan actitudes democráticas y de solidaridad que fomentan la inclusión social de toda la comunidad.

Esta transformación educativa que persigue el proyecto "Comunidades de aprendizaje" es posible gracias a la implementación de actuaciones educativas de éxito (AEE), aquellas prácticas educativas que han aportado evidencias de que contribuyen a promover el aprendizaje y a fomentar la cohesión social en todos los 
contextos en los que son implementadas (FLECHA, 2015), lo que implica que su transferibilidad a escenarios socioculturales, geográficos y económicos diversos queda garantizada. El proyecto que más ha contribuido a la consolidación de la investigación sobre comunidades de aprendizaje ha sido "INCLUD-ED": Strategies for inclusion and social cohesion in Europe from education (2006-2011), financiado por el 6. ${ }^{\circ}$ Programa Marco de la Comisión Europea. "INCLUD-ED" ha sido hasta la fecha una de las investigaciones científicas sobre educación en Europa de mayor amplitud, exhaustividad e impacto a nivel social, científico y político, siendo la única investigación de ciencias sociales seleccionada por la Comisión Europea entre las 10 historias de éxito en investigación europea. El proyecto contó con la participación de 15 instituciones que llevaron a cabo investigaciones en 14 países europeos distintos, con el objetivo de analizar qué prácticas educativas estaban contribuyendo a perpetuar las desigualdades en el plano europeo, y cuáles por su parte, estaban generando éxito educativo y mayores oportunidades para la inclusión y la cohesión social en una gran diversidad de contextos (FLECHA, 2015). Entre las actuaciones educativas de éxito identificadas por "INCLUD-ED" cabe destacar los grupos interactivos, las tertulias dialógicas, la formación de familiares, la participación educativa de la comunidad, el modelo dialógico de prevención y resolución de conflictos y la formación dialógica del profesorado.

\section{Transformación de centros educativos en comunidades de aprendizaje}

En la actualidad existen en el contexto español más de 200 escuelas constituidas en comunidades de aprendizaje en todos los niveles educativos, desde la educación infantil hasta la educación de personas adultas. La sostenibilidad de comunidades de aprendizaje en España, así como las contribuciones realizadas por el proyecto "INCLUD-ED", han hecho posible que las actuaciones educativas de éxito que conforman la

\section{CAMIIIAกกO JUחTOS}

¿Cómo puedo colaborar en la transformación de la escuela de mis hijos?

У Soñando la mejor escuela para todos los niños.

\ Implicándome en la gestión y organización del centro.

\ Participando como voluntario/a en actividades escolares o extraescolares.

У Ampliando mi formación en distintos campos, a partir de las iniciativas de formación de familiares.

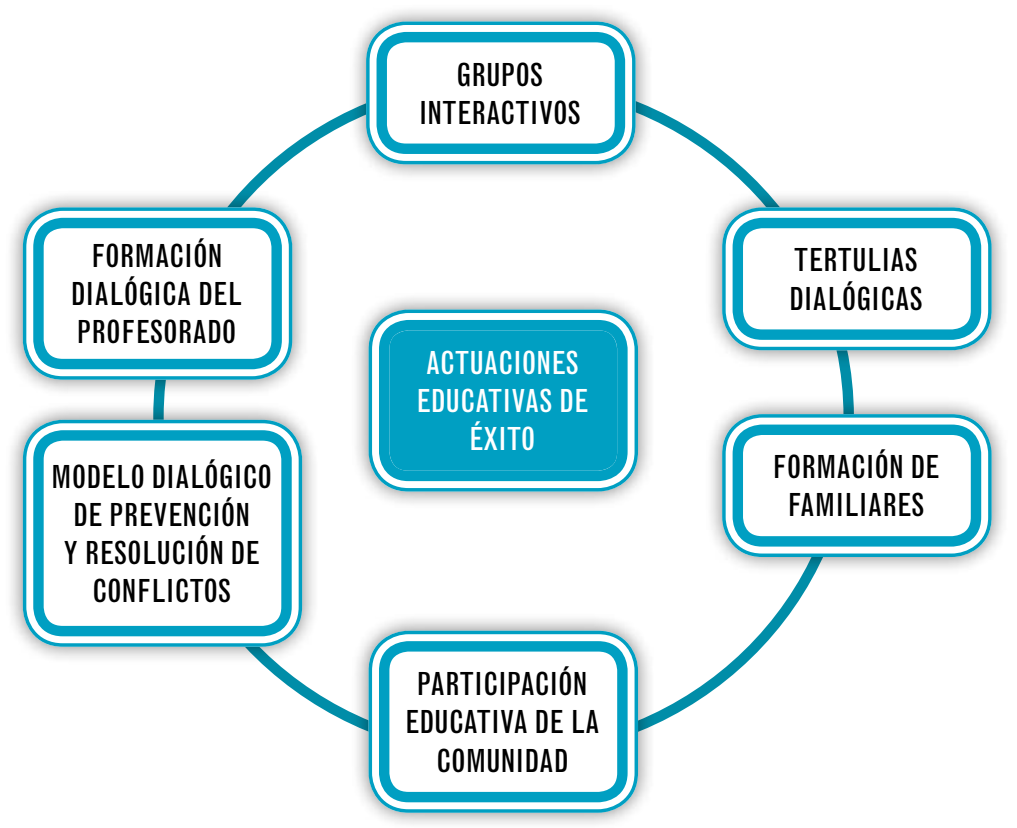

propuesta de "Comunidades de aprendizaje" se transfieran a numerosos centros educativos en diversos países en Europa y Latinoamérica.

Los centros educativos que en distintos territorios del mundo han optado por transformarse en comunidades de aprendizaje no responden a un perfil único de escuela, sino que encontramos una gran variedad de centros en entornos geográficos, socioeconómicos y culturales muy diversos, con distintas problemáticas, retos y motivaciones para iniciar el cambio (García et al., 2013). Sin embargo, sí podemos afirmar que todos los centros que optan por convertirse en una comunidad de aprendizaje comparten el objetivo común de mejorar los resultados del alumnado ofreciendo mayores oportunidades de éxito para todos, y promoviendo la cohesión social de toda la comunidad. Esta mejora de resultados se hace visible en el apren- 
Algunas de las principales características del proyecto de comunidades de aprendizaje son:

$\searrow$ Evidencias científicas: se implementan actuaciones educativas de éxito (AEE) que han demostrado científicamente que contribuyen a la mejora de resultados educativos y a la cohesión social.

$\checkmark$ Relevancia de las interacciones: en la actual sociedad de la información el aprendizaje del alumnado depende cada vez más de las interacciones que el alumnado tiene con todas las personas con las que se relaciona.

\ Participación de las familias y la comunidad: todos los agentes educativos pueden contribuir a soñar la escuela que desean y a participar en su construcción.

Fases del proceso de transformación en comunidades de aprendizaje

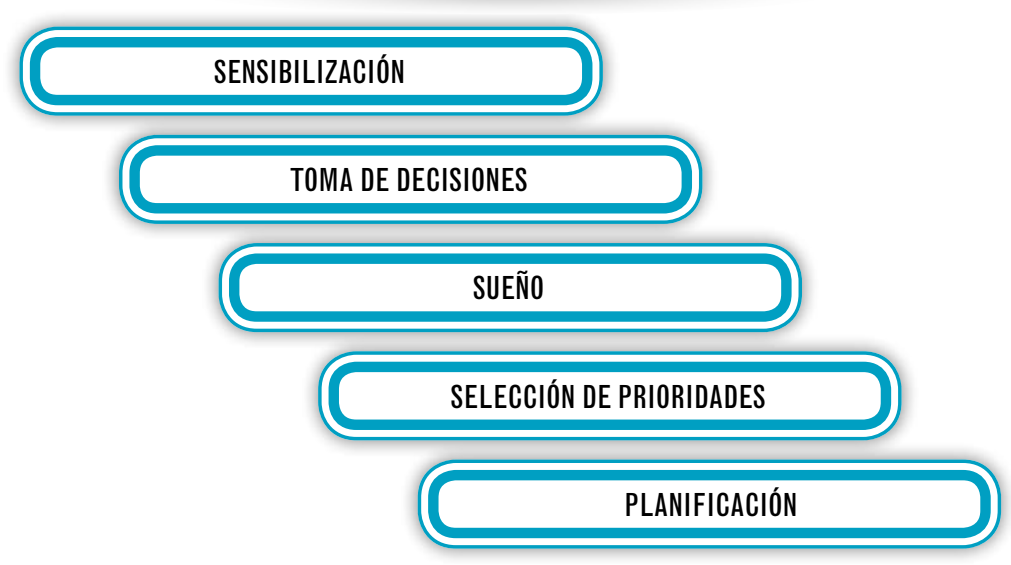

dizaje instrumental de los estudiantes, pero también en lo que respecta a sus valores, emociones y sentimientos.

Y toda esta gran transformación empieza con un sueño. El proceso de transformación de una escuela en comunidad de aprendizaje cuenta con las fases de

\section{DARA SABER MÂS}

Flecha, R. (2015). Successful Educational Actions for Inclusion and Social Cohesion in Europe. New York: Springer.

FREIRE, P. (1997). A la sombra de este árbol. Barcelona: El Roure.

García, C., Lastikka, A.L., \& Petreñas, C. (2013). Comunidades de aprendizaje. Scripta Nova - Revista Electrónica de Geografía y Ciencias Sociales, XVII (427). Recuperado de http://www.ub.edu/geocrit/sn/sn-427/sn-427-7.htm sensibilización, toma de decisiones, sueño, selección de prioridades y planificación. En la primera fase se dan a conocer las bases y principios de las comunidades de aprendizaje a todos los agentes educativos que, tras esta formación, deciden en la fase de toma de decisiones si quieren o no transformar su centro. En caso afirmativo, se da comienzo a la tercera fase: el sueño. Freire (1997), uno de los pedagogos más influyentes del siglo XX, dijo: "La educación, en verdad, necesita tanto de formación técnica, científica y profesional como de sueños y utopías". Siguiendo esta misma idea, en comunidades de aprendizaje se inicia el cambio invitando a soñar a todos los miembros de la comunidad educativa: alumnos, familias, profesores y demás agentes implicados imaginan la escuela que quieren para sí mismos, sus hijos, sus alumnos, etc.

A partir de los sueños expresados por todos los agentes implicados, se establecen las prioridades para alcanzarlos, y se comienza a transformar el centro educativo a partir de la participación de toda la comunidad educativa en la implementación de las actuaciones educativas de éxito. Una vez alcanzados los objetivos propuestos, se vuelve a soñar o se trabaja en la consecución de los siguientes objetivos por orden de prioridad. Soñar nunca es el fin, sino el medio para que se haga posible el lema que ilustra el proceso de transformación de todos estos centros educativos: "que el aprendizaje que queremos para nuestros hijos esté al alcance de todas las niñas y niños"•

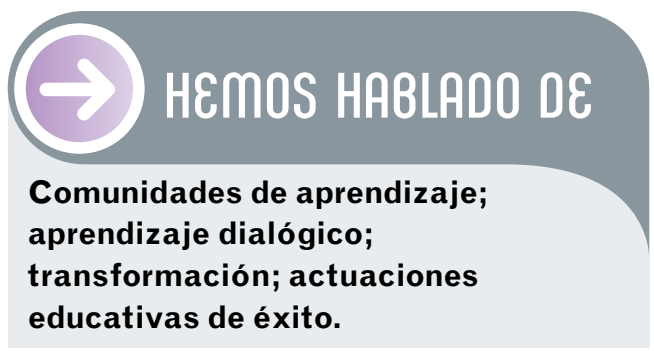

Este artículo fue solicitado por PADRES y MAESTROS en diciembre de 2015, revisado y aceptado en julio de 2016. 